\title{
EL INSIGHT EN ESQUIZOFRENIA DESDE EL MODELO DE ASIMILACIÓN: \\ UN ESTUDIO CUALITATIVO DE CASO A TRAVÉS \\ DE NARRACIONES
}

\section{THE INSIGHT IN SCHIZOPHRENIA FROM THE} ASSIMILATION MODEL:

\section{A QUALITATIVE CASE STUDY THROUGH NARRATIVES}

\section{Restituto Vañó Monllor}

Fundación General de la Universidad de Valencia. España

ORCID: https://orcid.org/0000-0002-0828-1922

\section{Isabel Caro Gabalda}

Facultad de Psicología. Departamento de Personalidad, Evaluación y Tratamientos Psicológicos.

Universidad de Valencia. España

\section{Sergio Pérez Ruiz}

Facultad de Psicología. Universidad Europea de Valencia. España

ORCID: https://orcid.org/0000-0001-8034-2198

Cómo referenciar este artículo/How to reference this article:

Vañó, R., Caro, I. y Pérez, S. (2020). El Insight en esquizofrenia desde el modelo de asimilación: Un estudio cualitativo de caso a través de narraciones. Revista de Psicoterapia, 31(117), 313-329. https:// doi.org/10.33898/rdp.v31i117.413

Fecha de recepción v1: 21-6-2020. Fecha de recepción v2: 25-8-2020. Fecha de aceptación: 28-9-2020. Correspondencia sobre este artículo:

E-mail: isabel.caro@uv.es

(C) 2020 Revista de Psicoterapia 


\title{
Resumen
}

El Insight es un elemento clave del proceso de asimilación, reflejando un nivel elevado y un progreso en la asimilación. En la esquizofrenia es, además, un elemento crucial relacionado con diferentes déficits metacognitivos. Por ello, la meta de este trabajo está en analizar, cualitativamente, los Insights alcanzados por un sujeto con esquizofrenia a lo largo de una serie de narraciones. Estos Insights, en un sujeto que no muestra un progreso en la asimilación de sus experiencias problemáticas, permiten reflexionar sobre el modelo de asimilación. Así, se plantea en la Discusión la necesidad de considerar a algunos de estos Insights, como "pseudo-insights" que no reflejarian, a diferencia de la formulación inicial del modelo, un progreso en la asimilación. Es decir, estos Insights no estarían centrados en resolver el problema, sino que podrían reflejar bajos niveles de Insight sobre la enfermedad y la dificultad para afrontar las emociones concurrentes. Podrían suponer una "capa de azúcar" sobre el problema en lugar de su afrontamiento para progresar en su asimilación. Se sugiere que diferenciar entre Insight y pseudo-Insight, y la función de cada uno de ellos, daría una mayor precisión al modelo, ayudándonos a entender el porqué de algunas dificultades en el proceso de asimilación.

Palabras clave: esquizofrenia, Insight, modelo de asimilación, APES, metacognición.

\begin{abstract}
Insight is a key element of the assimilation process, reflecting a high level and progress in assimilation. In schizophrenia, it is also a crucial element related to different metacognitive deficits. Therefore, the goal of this paper is to analyze, qualitatively, the Insights achieved by a subject with schizophrenia throughout a series of narratives. These Insights, in a subject who does not show progress in the assimilation of his problematic experiences, allow us to reflect on the assimilation model. Thus, the Discussion raises the need to consider some of these Insights, as "pseudo-insights" that would not reflect, unlike the initial formulation of the model, a progress in assimilation. In other words, these Insights would not be focused on solving the problem but could reflect low levels of Insight about the disease and the difficulty in coping with concurrent emotions. They could imply a "sugar coating" over the problem instead of coping with it to progress in its assimilation. It is suggested that differentiating between Insight and pseudo-Insight, and the function of each, would give greater accuracy to the model, helping us to understand the reasons of some difficulties in the assimilation process.
\end{abstract}

Keywords: Schizophrenia, Insight, assimilation model, APES, meta-cognition. 
El desarrollo de recursos personales con los que afrontar las diversas situaciones vitales que se nos presentan es uno de los aspectos que median en la resolución de conflictos por parte de las personas. En sujetos con esquizofrenia el desarrollo de estos recursos se ve condicionado por la existencia de sesgos cognitivos, de atribución, y otra serie de problemas metacognitivos que limitan su capacidad para resolver satisfactoriamente estas situaciones.

El modelo de asimilación es un modelo teórico acerca del proceso del cambio terapéutico (Stiles, 2002, 2011). La mayor parte de las investigaciones que se han hecho sobre este modelo se han centrado en analizar el cambio en pacientes diagnosticados con problemas depresivos y de ansiedad (Brinegar et al., 2006; Caro, 2003a, 2003b, 2011; Detert et al., 2006; Meystre et al., 2014; Osatuke et al., 2011a; Stiles, 2002), siendo muy escasos los estudios con pacientes esquizofrénicos (Osatuke et al., 2011b; Vañó y Caro, 2015).

Desde esta perspectiva, y teniendo en cuenta que el Insight es un elemento clave y complejo para el desarrollo del proceso de asimilación, consideramos especialmente interesante su estudio en un sujeto esquizofrénico. Como han señalado diversos autores, estos sujetos tendrán más dificultades para desarrollar y mantener un Insight (Lysaker et al., 2013; Nicolò et al., 2012; Osatuke et al., 2008; Phalen et al., 2015). Por ello, describir los Insights alcanzados por un sujeto con esquizofrenia nos va a permitir inferir algunas cuestiones relevantes para la aplicación del modelo de asimilación en este contexto clínico.

\section{El modelo de asimilación}

El modelo de asimilación permite explorar y describir el cambio, entendido éste como asimilación de experiencias problemáticas a través de una secuencia de estadios o niveles predecible (Stiles, 2002), uno de los cuales es el nivel de Insight. El modelo de asimilación de experiencias problemáticas es un modelo procesual, cualitativo e integrador, que permite entender el cambio en contextos terapéuticos (Caro et al., 2017).

Un concepto clave es el concepto de voz, lo que acerca el modelo de asimilación a perspectivas dialogales sobre el sí mismo (Hermans et al., 1992; Hermans y Dimaggio, 2004). Además, el concepto de voz es importante para entender el desarrollo del Insight, como expondremos más adelante.

El concepto de voz asume una perspectiva que, a través de la presencia de conversaciones internas, enfatiza la existencia de un sí mismo multifacetado que explica cómo las personas se comportan de forma aparentemente contradictoria en situaciones diferentes (Honos-Webb y Stiles, 1998). Las voces son trazos de experiencias anteriores que van configurando las diferentes representaciones que las personas tienen sobre el mundo y sobre ellos mismos. Estas voces van dando lugar a una comunidad de voces conectadas por significados compartidos que se activan ante características diferenciales de cada experiencia, con el fin de aportar diferentes recursos con los que afrontarla. De esa forma, el sí mismo se concibe 
como una comunidad de voces, donde destaca la naturaleza activa de los diferentes significados que se activan ante cada situación (Honos-Webb y Stiles, 1998). Por ejemplo, no emplearemos las mismas voces al intentar escribir un artículo científico, que una carta personal a un amigo.

Desde el modelo de asimilación, al introducir el término de 'voces', se distingue entre voces dominantes (VDs) y no dominantes (VNDs) haciendo referencia a la voz que forma parte de la comunidad de voces que conforman el sí mismo y a la voz problemática, respectivamente (Honos-Webb y Stiles, 1998).

Partiendo de esta base, hemos de entender que una voz problemática viene a reflejar una nueva experiencia o situación sobre la que una persona no tiene una forma adecuada (recurso) de afrontamiento. Es decir, ninguna voz de las que conforman el sí mismo dispone de una respuesta o pauta de acción con la que responder a dicha situación. Es por ello por lo que esta voz problemática lleva a la persona a experimentar "molestia”, "dolor”, "ansiedad”, "angustia” y otras emociones "negativas”, en tanto que la comunidad de voces dominante, que representa el sí mismo, no sabe cómo actuar adecuadamente. Por ejemplo, si siempre hemos tenido éxito en nuestro trabajo, nos va a ser difícil asimilar que nos hayan despedido de éste, y vamos a carecer, en principio, de recursos para afrontar dicha situación. Paralelamente al desarrollo del modelo de asimilación se elaboró la Escala de Asimilación de Experiencias Problemáticas (Assimilation of Problematic Experiences Scale - APES; Stiles et al., 1990, 1991) con los ocho niveles secuenciados del proceso de cambio. En el Cuadro 1 se desarrollan, brevemente, dichos niveles. Todos ellos suponen un distinto tipo de juego o diálogo entre voces (Stiles, 2011). En el inicio tenemos supresión de voces problemáticas (Niveles 0-Supresión y 1-Pensamientos no deseados). La aparición de la voz problemática (Nivel 2-Surgimiento), suele cursar con afecto elevado. Después de estos niveles inferiores, se pasa a los niveles intermedios. En el Nivel 3 (Clarificación), la experiencia problemática empieza a entenderse, aunque la comprensión del problema y de los aspectos relacionados con el mismo aparece, claramente, en el Nivel 4 (Insight). Una vez que se ha dado esta comprensión, se pasa a niveles de acción, como el Nivel 5 (Aplicación), o el 6 (Solución del Problema), hasta llegar al Nivel 7 (Dominio), en donde las voces problemáticas se integran, ya como recursos, en la comunidad de voces del sujeto.

Cuando los sujetos acceden a terapia, sus experiencias problemáticas suelen reflejar niveles inferiores de la APES (por regla general, niveles 1-Pensamientos no deseados, o 2-Surgimiento). Luego, con el trabajo terapéutico, se puede pasar a niveles que reflejan una mayor comprensión de la experiencia problemática, como el 3-Clarificación, y el 4-Insight, para, finalmente, alcanzar niveles elevados (APES-5, 6 y 7) (Stiles, 2001). No obstante, el proceso de asimilación, sobre todo en terapias de corte directivo, no es lineal, produciéndose avances y retrocesos entre los niveles APES (Caro y Stiles, 2013). 


\section{El papel del Insight}

Un elemento clave, en el contexto del modelo de asimilación, es la adquisición del Insight. En concreto, cuando hablamos de Insight hacemos referencia a un rico y complejo fenómeno planteado desde diferentes perspectivas teóricas (Kramer et al., 2016). En las personas con esquizofrenia este fenómeno es básico, habiéndose llegado a relacionar una pobre capacidad de Insight con baja conciencia de enfermedad, hospitalizaciones más frecuentes, mayores niveles de sintomatología positiva y negativa, menor funcionamiento psicosocial, actitudes negativas hacia la medicación y episodios más amplios de falta de adherencia a los antipsicóticos (Lysaker et al., 2011).

Visto de este modo, y desde el modelo de asimilación, el Insight es un componente crucial para la asimilación de experiencias problemáticas (Caro y Pérez, 2014). A través del Insight se establecen puentes de significado entre las VDs y las VNDs, siendo éstos relevantes en el proceso de asimilación. Tanto es así que, a partir del establecimiento de Insights (APES-4; véase Cuadro 1), se pueden diferenciar los casos de éxito y no éxito terapéutico (Brinegar et al, 2006; Detert et al., 2006; Honos-Webb et al., 1998).

\section{Cuadro 1. Escala de Asimilación de Experiencias Problemáticas}

0 Supresión/ Disociación. El cliente no es consciente del problema. El afecto implicado puede ser mínimo por efecto de una supresión realizada con éxito.

1 Pensamientos no deseados. Los clientes prefieren no pensar en la experiencia; los temas los plantea el terapeuta o circunstancias externas. El afecto contiene sensaciones/sentimientos sin definir y su conexión con el contenido no está clara.

2 Surgimiento/ Reconocimiento vago. El cliente reconoce la existencia de una experiencia problemática pero no puede formular el problema claramente. El afecto incluye intenso dolor psicológico o pánico asociado con los pensamientos y experiencias.

3 Clarificación/ Enunciar el problema. El contenido incluye un claro enunciado del problema - algo sobre lo que se estaría trabajando, o sobre lo que se podría trabajar. Las voces opuestas, diferenciadas, pueden hablar entre sí. El afecto es negativo pero manejable.

4 Comprensión/Insight. La experiencia problemática se expresa y comprende en alguna medida. El afecto puede ser mixto, reconociendo algunas molestias y aspectos agradables.

5 Aplicación/Elaboración. La comprensión se emplea en trabajar sobre el problema. El tono afectivo es positivo, serio, directo, optimista.

6 Solución del problema. El cliente consigue una solución adecuada para su problema específico. El afecto es positivo, de satisfacción, con orgullo por los logros alcanzados

7 Dominio/ Maestría. El cliente generaliza las soluciones a otras situaciones. El afecto es positivo, distinto y neutral (p.ej., no tiene capacidad para producir ninguna activación emocional).

Nota: La asimilación es considerada como un continuo, que permite niveles intermedios. Por ejemplo, 2.5 representa un nivel de asimilación a medio camino entre el Surgimiento (APES-2) y Enunciar o Clarificar el problema (APES-3).

Fuente: a partir de Honos-Webb y Stiles, 1998; Honos-Webb et al., 2003; Surko, et al., 1999; Stiles, et al., 1991; Stiles y Angus, 2001; Stiles, et al., 2004. 
Al mismo tiempo, el diálogo entre voces, o la asimilación, implica una perspectiva metacognitiva (Osatuke y Stiles, 2010). Así, si la elaboración de Insights queda dentro de los procesos metacognitivos, su desarrollo puede estar afectado de forma grave en la esquizofrenia (Lysaker et al., 2013; Nicolò et al., 2012; Osatuke et al., 2008; Phalen et al., 2015; Rabin et al., 2014).

En este sentido, se debe indicar que déficits importantes en la capacidad para reflexionar sobre uno mismo correlacionan con una memoria verbal, un Insight y una función social más deteriorada, menor conciencia emocional y paranoia (Dimaggio et al., 2010; Osatuke et al., 2008). Es más, Osatuke et al. (2008) señalan que un bajo nivel de Insight sobre la enfermedad puede explicarse etiológicamente como una defensa, en tanto que conlleva importantes funciones protectoras que de no existir podrían derivar en desmoralización, depresión, o incluso suicidio. Así, en los sujetos con una fuerte negación de su enfermedad, se aprecian correlaciones moderadas con bajos niveles de Insight. Además, suelen emplear con mayor frecuencia estrategias de reevaluación positiva y de evitación-escape (no focalizándose en el problema o involucrándose en ilusiones) (Lysaker et al., 2003).

En casos de personas con enfermedad mental sería aplicable la observación realizada por Kramer et al. (2016) acerca de que sus dificultades en la resolución de las experiencias problemáticas influyen en la integración de su identidad. Así, no sólo la dominancia entre las voces puede conllevar la falta de resolución de las experiencias traumáticas, a través de paradojas, sino que esta falta de resolución influye de forma circular reafirmando el posicionamiento de voces que impiden cualquier tipo de progreso. En este sentido, y siguiendo a Kramer et al. (2016), los procesos psicológicos afectados por estas experiencias pueden ser tan variados y cruciales, como "la calidad de las representaciones, los modelos de relación, las capacidades de metacognición, los esquemas interpersonales, la regulación del afecto, y la adherencia al tratamiento” (p. 144).

Atendiendo a todo ello, el foco en el Insight, en el contexto de un sujeto diagnosticado con esquizofrenia, se convierte en un aspecto importante desde la perspectiva del modelo de asimilación, para seguir construyendo teóricamente el modelo (Stiles, 2007). Por tanto, con este estudio cualitativo pretendemos reflejar las dificultades que experimenta un sujeto con esquizofrenia, a través de un estudio de carácter micro-nivel de los Insights alcanzados en su proceso de asimilación, a lo largo de diversas narraciones. Prestaremos una especial atención a algunos de los principales Insights alcanzados, analizándolos desde aquellos elementos, de tipo cognitivo, ya señalados y que dificultan la consecución de dichos Insights.

\section{Método}

\section{Participante}

El Sujeto X era un varón de 38 años, diagnosticado de esquizofrenia paranoide. Fue seleccionado por haber participado en una investigación anterior sobre 
el modelo de la asimilación. En este estudio, se le requirió aportar una serie de narraciones sobre "sus principales problemas". En ellas fue donde se autodenominó como Sujeto X (Vañó y Caro, 2015).

El sujeto presentaba diferentes fobias (a cruzar puentes, escaleras mecánicas, e ir en coche), así como ataques de miedo, ansiedad y apatía. Huérfano de madre, desde 2011, la relación con su padre no era muy buena por lo que vivía solo en un piso comprado con dinero ahorrado, bajo la supervisión económica de su hermano que hacía de tutor. El sujeto no trabajaba, aunque asistía a algunas asignaturas de un Ciclo Formativo de Grado Superior y a algunas actividades en un CRIS (Centro de Rehabilitación e Inserción Social). En cualquier caso, el sujeto cobraba una pensión que recibía su padre y le permitía asumir sus gastos cotidianos.

\section{Procedimiento de recogida de información}

El Sujeto X recibió tratamiento farmacológico por parte del psiquiatra y supervisión psicológica en el CRIS. Con el fin de interferir lo menos posible con los otros profesionales se acordó realizar una serie de sesiones de Counselling (13 en total, con un enfoque centrado en el cliente; Rogers, 1981) con una periodicidad semanal. Las sesiones las realizó el primer autor de este trabajo. El Sujeto X dio su consentimiento informado para emplear sus datos y sus narraciones para esta investigación.

Con el objetivo de analizar sus procesos de asimilación, se le pidió al sujeto que escribiera, tras cada una de estas sesiones, una narración semanal sobre un tema tratado en cada una de ellas con el fin de que hubiera una conexión con su día a día. Con el paso del tiempo el sujeto abandonó estas pautas, dejando de escribir semanalmente y atendiendo a temas que no estaban conectados con aspectos concretos de su experiencia diaria. Ante esta situación, se aceptaron estas narraciones como una muestra más de la expresión de su enfermedad mental por cuanto podían reflejar de forma más clara los conflictos entre las distintas voces que pudieran estar presentes. Las semanas en las que el sujeto no escribía las compensaba aportando dos narraciones en otro momento, de forma que se recogieron un total de 13 narraciones, algunas de ellas escritas el mismo día (las narraciones 2 y 3, y la 8 y 9), y cada una de ellas se tituló de manera distinta por el Sujeto X.

Se le administró, igualmente, una batería de medidas una semana antes de iniciar las sesiones de Counselling, y al finalizar las mismas. Esta batería estaba compuesta por las siguientes pruebas: HAD: Escala Hospitalaria de Ansiedad y Depresión (Zigmond y Snaith, 1971), BDI: Inventario para la Depresión de Beck (Beck et al., 1961), STAI: Inventario de Ansiedad Estado-Rasgo (Spielberger et al., 1970), SCL-90-R: Cuestionario de 90 síntomas (Derogatis, 1977), PANSS: Escala de los Síndromes Positivo y Negativo (Kay et al., 1987). Los resultados se pueden observar en la Tabla 1. Partiendo del cálculo del ICF (Índice de Cambio Fiable; Jacobson y Truax, 1991) podemos observar que no hubo cambio clínico entre el pretest y el postest. 
Tabla 1. Puntuaciones Pretest y Postest, y del Índice de Cálculo Fiable (ICF)

\begin{tabular}{|c|c|c|c|c|c|c|c|c|}
\hline & Pretest & Postest & $\begin{array}{l}\text { Puntos } \\
\text { de corte } \\
\text { clínicos }\end{array}$ & $\begin{array}{l}\text { Cambio } \\
\text { Clínico }\end{array}$ & $\begin{array}{l}\text { Diferencia } \\
\text { Cambio }\end{array}$ & ICF & $\begin{array}{c}\text { Cambio } \\
\text { estadísticamente } \\
\text { significativo }\end{array}$ & Interpretación \\
\hline BDI & 10 & 16 & 13.09 & $\mathrm{NO}$ & -6 & 1.25 & No & SC \\
\hline HAD-A & 12 & 15 & 8.83 & $\mathrm{NO}$ & -3 & 1.006 & No & $\mathrm{SC}$ \\
\hline HAD-D & 7 & 5 & 5.63 & $\mathrm{NO}$ & 2 & -0.65 & No & SC \\
\hline STAI-E & 60 & 80 & 24.94 & NO & -20 & 4.45 & Sí & Deterioro \\
\hline$\overline{\text { STAI-R }}$ & 80 & 87 & 28.36 & $\mathrm{NO}$ & -7 & 1.40 & No & SC \\
\hline$\overline{\mathrm{SOM}}$ & 0.6 & 0.75 & 0.93 & $\mathrm{NO}^{\star \star}$ & -0.15 & .15 & No & SC \\
\hline OBS & 2 & 2.5 & 0.87 & $\mathrm{NO}$ & -0.5 & 1.08 & No & SC \\
\hline INT & 1.33 & 2.44 & 0.88 & $\mathrm{NO}$ & -1.11 & 2.22 & Sí & Deterioro \\
\hline DEP & 1.76 & 2.76 & 1.02 & $\mathrm{NO}$ & -1 & 2.17 & Sí & Deterioro \\
\hline ANS & 2.4 & 3 & 0.89 & $\mathrm{NO}$ & -0.6 & 1.42 & No & SC \\
\hline $\mathrm{HOS}$ & 0.33 & 1 & 0.668 & $\mathrm{NO}$ & -0.66 & 1.26 & No & SC \\
\hline FOB & 1.28 & 1.57 & 0.44 & $\mathrm{NO}$ & -0.29 & 0.52 & No & SC \\
\hline PAR & 0.66 & 3 & 0.74 & NO & -2.34 & 3.75 & Sí & Deterioro \\
\hline $\mathrm{PSI}$ & 0.9 & 2.2 & 0.4 & $\mathrm{NO}$ & -1.3 & 2.93 & Sí & Deterioro \\
\hline$\overline{\mathrm{TGSI}}$ & 1.32 & 2.16 & 0.79 & $\mathrm{NO}$ & -0.84 & 3.81 & Sí & Deterioro \\
\hline PANSS(+) & 21 & 20 & 6.04 & $\mathrm{NO}$ & 1 & -22 & No & SC \\
\hline PANSS (-) & 16 & 16 & 8.67 & $\mathrm{NO}$ & 0 & 0 & No & SC \\
\hline $\begin{array}{l}\text { PANSS } \\
\text { (TOTAL) }\end{array}$ & 36 & 38 & 18.74 & NO & 2 & .32 & No & SC \\
\hline \multicolumn{9}{|c|}{$\begin{array}{l}\text { * Valores de ICF }>1.96 \text { resultan estadísticamente significativos para } p<0.05 \text {. A medida } \\
\text { que se incrementa el valor de la } \mathrm{RCl} \text { menor es la probabilidad de que el cambio } \\
\text { observado sea debido al azar. } \\
\text { * Por debajo del punto de corte crítico, tanto en el Pretest como en el Postest. }\end{array}$} \\
\hline
\end{tabular}

\section{Procedimiento de estudio del proceso de asimilación}

No se utilizó el modelo de la asimilación para planificar o conducir las sesiones de Counselling, sino que se aplicó posteriormente para comprender el proceso de comunicación entre las voces del sujeto, lo que ejemplificaba su proceso de asimilación. Tras la recogida del material, los dos principales investigadores (autores uno y dos de este trabajo) se centraron en el análisis entre las voces siguiendo los diferentes pasos propuestos por Stiles y Angus (2001): familiarización e indexación, identificación y elección de un tema, selección de pasajes y búsqueda de voces, y descripción del proceso de asimilación. Del proceso se obtuvieron un total de 85 fragmentos, o pasajes en los que se diferenciaron 6 voces (3 Dominantes: Seguridad, Sentido de Vida y Enfermedad mental. Y, 3 No Dominantes: Caos, Crisis de fe y Estigma), algunas de ellas con muy poca presencia, así como diferentes temas. Los principales temas del Sujeto X que aparecieron en sus narraciones fueron: El 
centro del mundo, Amor, Enfermedad mental, Mis relaciones y Yo en el mundo.

En todo este proceso trabajaron los dos investigadores principales de forma iterativa, sobre la base del Método Ward (Schielke et al., 2009).

Tras alcanzar un alto nivel de acuerdo entre estos investigadores principales se planteó introducir otro juez (el tercer autor) que permitiera contrastar los acuerdos obtenidos. En este paso se observaron divergencias entre algunas de las codificaciones de los dos investigadores principales y el tercer juez. Estas divergencias se fueron analizando y resolviendo en una serie de reuniones conjuntas con la vista puesta en lograr un acuerdo total entre jueces.

\section{Resultados}

\section{Descripción general del proceso de asimilación a lo largo de las narraciones}

Los datos obtenidos a lo largo de las narraciones aportadas por el sujeto nos ofrecen una serie de fragmentos puntuados en los niveles 2 . Reconocimiento vago (33\%), 3. Enunciado del problema (24\%) e Insight (24\%), así como un 5\% de puntuaciones en el nivel 1. Pensamientos No Deseados, y un 14\% en el nivel 5. Solución del Problema. Por otra parte, no se encontraron puntuaciones en los niveles 0. Evitación, 6. Solución del problema y 7. Dominio. De manera que podemos asumir que el Sujeto $\mathrm{X}$ se mueve, a lo largo de sus narraciones, en niveles bajos e intermedios de asimilación.

\section{Análisis cualitativo de los niveles de Insight}

Vamos a mostrar algunos de los Insights alcanzados por el Sujeto X. Analizaremos aquellos párrafos donde el sujeto atiende a su enfermedad mental, su sintomatología y problemas asociados, llegando, incluso, a asumir el papel de enfermo mental. Este es un tema fundamental en un sujeto con esquizofrenia. Describiremos dichos Insights desde el modelo de asimilación y desde diferentes procesos cognitivos, típicos, en un paciente con esquizofrenia.

Un punto de inicio puede ser los Insights que hacen referencia a su propia enfermedad. En este sentido, el sujeto se define a sí mismo, en la narración 3, que tituló "Mi Ansiedad", como un "Enfermo Mental”, lo que le lleva a asumir el rol de enfermo.

"Yo soy enfermo mental y tengo una esquizofrenia. Cada enfermo mental es Único. Mi enfermedad mental se caracteriza por tener delirios de grandeza o ideas extravagantes y emociones descontroladas. Ahora las emociones descontroladas que tengo son ansiedad y fobias, y los delirios me los estoy replanteando ante mi nueva situación. (4. Insight)”

Conviene señalar que una de las voces dominantes principales, en las narraciones, se denominó: "Enfermedad mental”. Desde ella, el sujeto atiende la sintomatología como un aspecto externo a él que en ocasiones experiencia, aunque en ocasiones tenía cierta capacidad de posicionarse sobre el resto de voces. Esta 
externalización de la enfermedad conlleva ciertas implicaciones, como no asumir ningún papel agente en su enfermedad, lo que puede dificultar progresar en la asimilación de sus problemas. Esto nos aparece en el siguiente fragmento, tomado también de la narración 3:

"Parece que a mi ansiedad le afecta el estrés indirectamente, pues a mayor estrés más frecuencia. También parece que ésta evoluciona pues no siempre es la misma" (4. Insight).

En el siguiente fragmento (narración 11) el sujeto sigue ahondando en el mismo aspecto, centrándose más en la sintomatología, y señalando más claramente que el problema y la solución es algo externo a él.

"Yo desde el 2014 he desarrollado una serie de fobias injustificadas desde un punto de vista ingenuo. Tengo miedo de las escaleras mecánicas, de cruzar puentes, de que me den miedo los coches mientras estoy subido a uno de ellos, de los viajes. Eso hace que las escaleras normales las utilice con apuro. Los puentes no los cruzo, no me puedo montar en los coches y no voy de viaje. Lo que ocurre es que no sé qué es lo que lo causa y no creo que la exposición gradual sirva de nada (2. Reconocimiento vago). Parece que hay algo mal en la química de mi cerebro que me hace sentir miedo y hasta que se arregle seguiré sintiendo miedo" (4. Insight).

En este último fragmento podemos observar como el Insight, "Parece que hay algo mal en la química de mi cerebro que me hace sentir miedo y hasta que se arregle seguiré sintiendo miedo" se produce justo después de una expresión que denota un nivel 2, de Reconocimiento vago: "Lo que ocurre es que no sé qué es lo que lo causa y no creo que la exposición gradual sirva de nada”. En cierta forma, este salto, de un nivel 2 a un nivel 4 de la APES, nos señala un mecanismo de "salto a las conclusiones" que el sujeto utiliza con relativa frecuencia en sus narraciones. Como podemos interpretar, ante una Voz No Dominante (VND) de Caos, la Voz Dominante (Enfermedad Mental) asume parte de ella y expone su visión con el fin de aportarle cierta disminución de esos aspectos negativos, de no saber qué es lo que lo causa.

Si seguimos atendiendo a otros Insights sobre el tema de la enfermedad mental, podemos analizar el siguiente fragmento (narración 11):

"Tengo ataques de ansiedad y cuando los tengo todo se paraliza para mí y empiezo a pensar negativamente de todo, a toda velocidad, y me desespero (3. Enunciado del problema). Los tengo desde 2009, y suelen ser habitualmente por las tardes, desde 2015 aparecieron nuevas formas de parálisis y suelen suceder sin que haya causa, o habiéndola parece insuficiente para producir tan devastador sentimiento. Esto hace que yo no sea previsible, pues si me comprometo y me da un ataque, habré de quedarme paralizado y renunciar a mi compromiso (4. Insight). También hace que no pueda alejarme demasiado de un sitio donde me pueda quedar paralizado de una manera tranquila. En un autobús o un tren no es 
aceptable. Por lo que no puedo irme de excursión pues corro el riesgo de quedarme con una angustia que me muero."

Aquí podemos observar nuevamente cómo el sujeto alcanza un Insight tras la expresión de una VND que relata su sintomatología. Ante esta VND, la VD de Enfermedad Mental se hace con el control de la situación expresando las consecuencias que tiene, en su vida diaria, su enfermedad mental. Como podemos observar, esta VD intenta normalizar esas consecuencias, y de esa forma parece asumir la realidad que le rodea; aunque en realidad no ha entrado a valorar otras posibilidades diferentes que el experimentarlas de forma pasiva, como algo que le sucede y sobre lo que no tiene control. Por ello, sigue asumiendo un rol de enfermo y, se puede inferir, que el Insight facilita reducir la tensión generada por la expresión de dicha sintomatología.

En el siguiente ejemplo (tomado de la narración 13), podemos ver cómo la VD de Enfermedad Mental ofrece sus planteamientos para aferrarse con determinación a su visión acerca de su experiencia. Aunque asume la posibilidad de que las VNDs (Caos) estén ofreciéndole una información más adaptativa, o real, de su experiencia, la VD se impone en su criterio, aunque aceptando la posibilidad de que pueda estar equivocada en su planteamiento.

"Yo tengo una enfermedad mental. Que se traduce en ideas raras, emociones descontroladas y marginación social. Yo creo sin ningún rigor científico, que mi enfermedad está ligada a mi inteligencia y que si quería ser un genio como el que quiero ser tendría que pasar por ella. Las ideas raras, aun equivocadas, son hipótesis de mi tremenda inteligencia. Las emociones descontroladas son producto de un cerebro que se sale, y mi marginación es consecuencia de mis rarezas. Yo tengo que triunfar con la enfermedad que no controlo, aunque si es como pienso que es, lo conseguiría con grandes pero vencibles dificultades. Pero si no es como pienso que es, podría hacerme imposible el éxito e incluso la vida, pues bastaría con que ciertos síntomas no desapareciesen" (4 Insight).

Este fragmento es importante también por cuanto nos permite apreciar que la VD, que se activa ante los síntomas y otros aspectos de su enfermedad, es una voz que a su vez limita el progreso del sujeto hacia su resolución; al no permitirle atender a las VNDs que le indican que algo no está conforme a la realidad, y que necesita un mejor ajuste para disponer de recursos para tratar con ella. A su vez, podemos, de alguna manera, entrever que dicha voz cumple ciertas funciones protectoras de su sí mismo. Aunque pueda ser sobre una base irreal, le permite posicionarse como alguien especial en un entorno donde sus refuerzos positivos son mínimos. Es por ello por lo que consideramos la función protectora de dichos Insights que, a su vez, pueden suponer dificultades en el proceso de asimilación de sus experiencias problemáticas. 


\section{Discusión}

Si bien, compartimos la propuesta de Osatuke et al. (2011b) acerca de que el modelo de asimilación parece especialmente adecuado para el espectro de la esquizofrenia, consideramos que lo que éste puede aportar al modelo de asimilación va más allá de la confirmación del mismo.

Creemos importante señalar que el análisis de casos de fracaso terapéutico es particularmente importante cara a las generalizaciones que se puedan hacer (Caro, 2006), en tanto que cabe preguntarnos qué detalles pueden aportar al modelo de asimilación y cómo éstos pueden estimular su desarrollo sobre la perspectiva de los estudios de caso que construyen una teoría (Stiles, 2007).

En este trabajo hemos presentado el caso de un sujeto con diagnóstico de esquizofrenia que no mostró una mejoría en la resolución de sus experiencias problemáticas, aunque, en determinados momentos mostró niveles altos de asimilación, como niveles APES-4, o Insights, sobre los que cabe hacer una serie de consideraciones.

Los Insights alcanzados por el Sujeto X cumplían los requisitos formales, dentro del modelo de asimilación, como para ser considerados como tales (Stiles y Angus, 2001). Es decir, la historia que cuenta el Sujeto X en sus narraciones muestra diversos puentes de significado, representando y acogiendo satisfactoriamente sus VDs y sus VNDs (Osatuke et al., 2004). Sin embargo, estos Insights se veían “matizados" por determinados procesos cognitivos, tal y como hemos descrito en los Resultados. Esto nos lleva a plantearnos que el modelo de asimilación podría desarrollarse de forma más precisa para poder encuadrar mejor los resultados obtenidos por sujetos que pueden presentar déficits a diferentes niveles: metacognitivos, salto a las conclusiones, etc. En este sentido, el nivel de asimilación en donde se podría apreciar mejor dichos déficits (y a falta de estudios posteriores) podría ser el nivel de Insight. Es en este nivel (véase Cuadro 1) donde las voces dialogan entre sí, y se alcanza una cierta comprensión sobre, por ejemplo, por qué me pasa lo que me pasa, por qué actúo de la manera que lo hago, etc. Esto nos aparece en los Insights mostrados en los Resultados, como, por ejemplo, el siguiente: "Parece que a mi ansiedad le afecta el estrés indirectamente, pues a mayor estrés más frecuencia. También parece que ésta evoluciona pues no siempre es la misma”. El Insight, por tanto, es clave en el proceso de asimilación de cualquier sujeto, aunque en el caso de pacientes con esquizofrenia se deberían hacer determinadas consideraciones que suponen contemplar este nivel de asimilación desde una perspectiva diferente, sugiriendo adaptaciones en la APES.

Así, los datos obtenidos parecen mostrar cierto apoyo a las conclusiones presentadas por Osatuke et al. (2008), en cuanto que el Insight podría tener cierta función de defensa psicológica; lo que podría ser causa, o consecuencia, de los déficits metacognitivos observados en el sujeto. Desde nuestro punto de vista, muchos de los Insights alcanzados por el sujeto podrían suponer una técnica para minimizar el malestar sobre el problema, alcanzando un Insight circunstancial que 
emerge de enfocar la atención en un aspecto relacionado o periférico al problema (por ejemplo, véase el primer ejemplo de la narración 11). En este sentido, se podría considerar que los Insights alcanzados por el Sujeto X supusieron poner, como señalaron Honos-Webb et al., (2000) una “capa de azúcar” en lugar de alcanzar una comprensión significativa de sus experiencias.

Por ello, en tanto que no podemos dejar de considerar los planteamientos del sujeto como Insights que se producían dentro del proceso de asimilación de su experiencia, tampoco podemos ignorar la posibilidad de que su incapacidad de resolución de sus experiencias problemáticas le haya llevado a reafirmar los posicionamientos de sus voces de forma tal que se haya situado en posiciones rígidas que le llevan a centrarse en unas pocas problemáticas sin alcanzar un resultado deseado (Kramer et al., 2016). En este sentido, si bien el sujeto logra algún Insight de tipo clínico (Lysaker et al., 2013; Phalen et al., 2015) al asumir la existencia de un trastorno y su diagnóstico, no parece distanciarse lo suficiente para realizar una evaluación precisa de sus propias creencias e interpretaciones, centrándose en aspectos circunstanciales a sus experiencias problemáticas. Esto es importante puesto que el Insight, como puente de significado, facilita el paso a niveles más elevados de asimilación. En consecuencia, con este tipo de sujetos, lo que formalmente se puede considerar un Insight, pero, experiencialmente, no lo es, supondría intentar entender o integrar otros procesos cognitivos en la propia asimilación, adaptando el modelo a características de los sujetos.

Así, planteamos que sería interesante que el modelo de asimilación permitiera diferenciar diferentes tipos de Insight, los centrados en el problema, que potencian la asimilación de las experiencias problemáticas, y aquellos centrados en la emoción y realizados sobre aspectos relacionados o periféricos al problema; que podemos diferenciar con el nombre de 'pseudo-Insights' y de los que no se deriva un progreso por el continuo de la asimilación; ya que en ningún momento se centra en resolver el problema. De hecho, estos pseudo-Insights podrían estar relacionados con bajos niveles de Insight sobre la enfermedad(Osatuke et al., 2008), principalmente derivados de la dificultad de afrontar las emociones emergentes al atender a la misma. Por todo ello, la diferenciación entre los insights y los pseudoinsigths permitiría dotar de una mayor precisión al modelo, evitando resultados aparentemente paradójicos al ubicar el tipo de insight en su nivel correspondiente de asimilación según la función que realiza.

Centrar la atención en estos "pseudo-Insights", nos puede permitir atender a las dificultades o resistencias del sujeto a enfrentarse a su experiencia problemática, siendo un mecanismo que permitiría explicar la falta de resolución de estas experiencias. Igualmente, nos podría ayudar a explicar por qué, en ocasiones, no se consigue un avance en la asimilación de sus experiencias problemáticas pese a que el sujeto obtiene diferentes valoraciones de Insight. Es más, consideramos que habría que plantearse estos pseudo-Insights como un marcador de niveles bajos de asimilación; en tanto que su función como defensa impide a los individuos el 
progreso en su resolución de los problemas, e incluso de alguna manera les puede impulsar a un nivel muy bajo de conciencia sobre el problema.

En cualquier caso, los datos mostrados son también un buen ejemplo de cómo las mismas dificultades del sujeto para atender a sus VND le derivan a una reevaluación positiva de la situación, así como a una estrategia de evitación y escape (Lysaker et al., 2003). Si bien estos ejemplos son sólo una muestra, estos mecanismos podemos hallarlos en otros párrafos de la muestra total de narraciones, donde el sujeto muestra su visión de la enfermedad como algo externo a él, o donde indica las precauciones que toma para no encontrarse en ninguna situación incómoda, etc.

\section{Limitaciones y estudios futuros}

En primer lugar, somos conscientes de que en este trabajo sólo esbozamos algunos aspectos que intervienen en este fracaso de asimilación de las experiencias problemáticas del Sujeto X. Definimos únicamente la existencia de Insights que no acompañan dicho proceso, y esbozamos tentativamente algunos de los aspectos observados. Nuestra intención es desarrollar posteriormente más elementos presentes en las narraciones, que evidencian dificultades metacognitivas del sujeto,en conjunción con resultados de otras investigaciones realizadas en el contexto de la esquizofrenia (Dimaggio et al., 2010; Jørgensen et al., 2015; Lysaker et al., 2003, 2013; Nicolò et al., 2012; Osatuke et al., 2008; Phalen et al., 2015).

Además, cabe resaltar que es un estudio de caso, sobre un sujeto con esquizofrenia, y la evidencia ha de circunscribirse, de momento, a estos datos. En cualquier caso, el estudio del proceso de asimilación en casos de personas con trastorno mental consideramos que es un campo que hay que seguir explorando, haciendo hincapié en aquellos donde no se ha obtenido una asimilación de las experiencias problemáticas, en tanto que pueden aportar información relevante para poder detallar el modelo de forma más precisa. Por ejemplo, considerar un pseudo-Insight como un marcador de baja asimilación que abre la puerta a integrar procesos cognitivos, especialmente relevantes en el contexto de la esquizofrenia, a la hora de evaluar el proceso de asimilación de experiencias problemáticas.

Entendemos que una de las limitaciones más importantes es el no contar con una evaluación metacognitiva del sujeto realizada previamente al registro de las narraciones, siendo interesante la adaptación de herramientas ya desarrolladas por otros investigadores, como la Metacognition Assessment Scale (Semerari et al., 2003). En este sentido, consideramos que la citada escala podría aplicarse igualmente a las narraciones, y que la información que nos aportaría podría identificar estas carencias a nivel de metacognición, relacionándolas con progresos en la APES.

También se podría considerar el hecho de que el sujeto haya participado previamente en otra investigación, pese a que se cuidó que no tuviera acceso a los resultados de la misma y se mantuviera una distancia entre la recogida de información y el modelo a emplear en su análisis.

Por otro lado, el formato de recogida de la información utilizado en esta in- 
vestigación, a través de narraciones, también supone una limitación ya que, en el sujeto, el diálogo entre las voces se ve matizado por una escritura en la que puede haberse plasmado un contenido más estático, careciendo de una mayor flexibilidad propia del intercambio entre el terapeuta y el cliente.

A pesar de estas limitaciones, creemos que estudios de este tipo, con sujetos con estas características nos permiten avanzar y desarrollar los planteamientos principales del modelo de asimilación y su relevancia para describir aspectos importantes para el cambio terapéutico.

\section{Referencias}

Beck, A. T., Ward, C. H., Mendelson, M., Mock, J. y Erbaugh, J. (1961). An inventory for measurement of depression. Archives of General Psychiatry, 4(6), 561-571.https://doi.org/10.1001/archpsyc.1961.01710120031004

Brinegar, M. G., Salvi, L. M., Stiles, W. B. y Greenberg, L. S. (2006). Building a meaning bridge: Therapeutic progress from problem formulation to understanding. Journal of Counseling Psychology, 53(2), 165-180. https://doi.org/10.1037/0022-0167.53.2.165

Caro, I. (2003a). La resolución de experiencias problemáticas en la terapia lingüística de evaluación según el modelo de asimilación (I). Boletín de Psicología, 79, 31-58.

Caro, I. (2003b). La resolución de experiencias problemáticas en la terapia lingüística de evaluación según el modelo de asimilación. El análisis de Silvia (II). Boletín de Psicología, 79, 59-84.

Caro, I. (2006). The assimilation of problematic experiences in linguistic therapy of evaluation: How did Maria assimilate the experience of dizziness? Psychotherapy Research, 16(4), 422-435. https://doi. org/10.1080/10503300600756436

Caro, I. (2011). El cambio terapéutico a través del modelo de asimilación: su aplicación en la terapia lingüística de evaluación. Revista de Psicopatología y Psicología Clínica, 16(3), 169-188. https://doi.org/10.5944/ rppc.vol.16.num.3.2011.10360

Caro, I. y Pérez, S. (2014). La asimilación de experiencias problemáticas: el diálogo entre voces en el nivel de Insight. Revista de Psicoterapia, 25(99), 83-96.

Caro, I., Pérez, S. y Vañó, R. (2017). El modelo de asimilación como un modelo integrador sobre el cambio terapéutico. Revista de Psicoterapia, 28(108), 105-120. https://doi.org/10.33898/rdp.v28i108.204

Caro, I. y Stiles, W. B. (2013). Irregular assimilation progress: Reasons for setbacks in the context of linguistic therapy of evaluation. Psychotherapy Research, 23(1),35-53.https://doi.org/10.1080/10503307.2012.721938

Derogatis, L. R. (1977). SCL-90-R. Symptom checklist 90 revised. NCS Pearson.

Detert, N. B., Llewelyn, S., Hardy, G. E., Barkham, M., y Stiles, W. B. (2006). Assimilation in good-and pooroutcome cases of very brief psychotherapy for mild depression: An initial comparison. Psychotherapy Research, 16(4), 393-407. https://doi.org/10.1080/10503300500294728

Dimaggio, G., Hermans, H. J. y Lysaker, P. H. (2010). Health and adaptation in a multiple Self: The role of absence of dialogue and poor metacognition in clinical populations. Theory \& Psychology, 20(3), 379-399. https:// doi.org/10.1177\%2F0959354310363319

Hermans, H. J. y Dimaggio, G. (2004). The dialogical self in psychotherapy: An introduction. En H.J. Hermans y G. Dimaggio (eds.), The dialogical self in psychotherapy (pp. 1-12). Brunner-Routledge.

Hermans, H. J., Kempen, H. J. y Van Loon, R. J. (1992). The dialogical self: Beyond individualism and rationalism. American Psychologist, 47(1), 23-33. https://doi.org/10.1037/0003-066X.47.1.23

Honos-Webb, L., Harrick, E., Stiles, W. B. y Park, C. (2000). Assimilation of traumatic experiences and physicalhealth outcomes: Cautions for the Pennebaker paradigm. Psychotherapy, 37(4), 307-314. https://doi. org/10.1037/0033-3204.37.4.307

Honos-Webb, L. y Stiles, W. B. (1998). Reformulation of assimilation analysis in terms of voices. Psychotherapy: Theory, Research, Practice, Training, 35(1), 23-33. https://doi.org/10.1037/h0087682

Jacobson, N. S. y Truax, P. (1991). Clinical significance: a statistical approach to defining meaningful change in psychotherapy research. Journal of Consulting and Clinical Psychology, 59(1), 12-19. https://doi. org/10.1037/0022-006X.59.1.12 
Jørgensen, R., Licht, R. W., Lysaker, P. H., Munk-Jørgensen, P., Buck, K. D., Jensen, S. O. W., Hansson, L. y Zoffmann, V. (2015). Effects on cognitive and clinical insight with the use of guided Self-determination in outpatients with schizophrenia: a randomized open trial. European Psychiatry, 30(5), 655-663. https:// doi.org/10.1016/j.eurpsy.2014.12.007

Kay, S. R., Fiszbein, A. y Opfer, L.A. (1987). The positive and negative syndrome scale (PANSS) for schizophrenia. Schizophrenia Bulletin, 13(2), 261-276. https://doi.org/10.1093/schbul/13.2.261

Kramer, U., Meystre, C., Imesch, C. y Kolly, S. (2016). Assimilative processes in a client with borderline personality disorder: Tracking internal multiplicity over the first ten sessions of therapy. Journal of Psychotherapy Integration, 26(2), 144-159. https://doi.org/10.1037/a0040044

Lysaker, P. H., Bryson, G. J., Lancaster, R. S., Evans, J. D. y Bell, M. D. (2003). Insight in schizophrenia: associations with executive function and coping style. Schizophrenia research, 59(1), 41-47. https://doi. org/10.1016/S0920-9964(01)00383-8

Lysaker, P. H., Dimaggio, G., Buck, K. D., Callaway, S. S., Salvatore, G., Carcione, A., Nicolò, G. y Stanghellini, G. (2011). Poor insight in schizophrenia: links between different forms of metacognition with awareness of symptoms, treatment need, and consequences of illness. Comprehensive Psychiatry, 52(3), 253-260. https://doi.org/10.1016/j.comppsych.2010.07.007

Lysaker, P. H., Vohs, J., Hillis, J. D., Kukla, M., Popolo, R., Salvatore, G. y Dimaggio, G. (2013). Poor insight into schizophrenia: contributing factors, consequences and emerging treatment approaches. Expert Review of Neurotherapeutics, 13(7), 785-793. https://doi.org/10.1586/14737175.2013.811150

Meystre, C., Kramer, U., De Roten, Y., Despland, J. N. y Stiles, W. B. (2014). How psychotherapeutic exchanges become responsive: A theory-building case study in the framework of the assimilation model. Counselling and Psychotherapy Research, 14(1), 29-41. https://doi.org/10.1080/14733145.2013.782056

Nicolò, G., Dimaggio, G., Popolo, R., Carcione, A., Procacci, M., Hamm, J., Buck, K., Pompili, E., Buccione, I., Lagrotteria, B. y Lysaker, P. H. (2012). Associations of metacognition with symptoms, insight, and neurocognition in clinically stable outpatients with schizophrenia. The Journal of Nervous and Mental Disease, 200(7), 644-647. https://doi.org/10.1097/NMD.0b013e31825bfb10

Osatuke, K., Ciesla, J., Kasckow, J. W., Zisook, S. y Mohamed, S. (2008). Insight in schizophrenia: a review of etiological models and supporting research. Comprehensive Psychiatry, 49(1), 70-77. https://doi. org/10.1016/j.comppsych.2007.08.001

Osatuke, K. y Stiles, W. B. (2010). Change in post traumatic stress disorder: An assimilation model account. En G. Dimaggio y P. H. Lysaker (eds.), Metacognition and severe adult mental disorders: From research to treatment (pp. 285-300). Brunner-Routledge.

Osatuke, K., Stiles, W. B., Barkham, M., Hardy, G. E. y Shapiro, D. A. (2011a). Relationship between mental states in depression: The assimilation model perspective. Psychiatry Research, 190(1), 52-59. https://doi. org/10.1016/j.psychres.2010.11.001

Osatuke, K., Reid, M., Stiles, W. B., Kasckow, J. W., Zisook, S. y Mohamed, S. (2011b). Narrative evolution and assimilation of problematic experiences in a case of pharmacotherapy for schizophrenia. Psychotherapy Research, 21(1), 41-53. https://doi.org/10.1080/10503307.2010.508760

Phalen, P. L., Viswanadhan, K., Lysaker, P. H. y Warman, D. M. (2015). The relationship between cognitive insight and quality of life in schizophrenia spectrum disorders: Symptom severity as potential moderator. Psychiatry Research, 230(3), 839-845. https://doi.org/10.1016/j.psychres.2015.10.014

Rabin, S. J., Hasson-Ohayon, I., Avidan, M., Rozencwaig, S., Shalev, H. y Kravetz, S. (2014). Metacognition in schizophrenia and schizotypy: relation to symptoms of schizophrenia, traits of schizotypy and social quality of life. Israel Journal of Psychiatry and Related Sciences, 51(1), 44-53.

Rogers, C. R. (1981). La psicoterapia centrada en el cliente. Paidós.

Schielke, H. J., Fishman, J. L., Osatuke, K. y Stiles, W. B. (2009). Creative consensus on interpretations of qualitative data: The Ward method. Psychotherapy Research, 19(4-5), 558-565. https://doi. org/10.1080/10503300802621180

Semerari, A., Carcione, A., Dimaggio, G., Falcone, M., Nicolò, G., Procacci, M. y Alleva, G. (2003). How to evaluate metacognitive functioning in psychotherapy? The Metacognition Assessment Scale and its applications. Clinical Psychology \& Psychotherapy, 10(4), 238-261. https://doi.org/10.1002/cpp.362

Spielberger, C. D., Gorsuch, R. L. y Lushene, R. E. (1970). STAI, Manual for the State-Trait Anxiety Inventory (SelfEvaluation Questionnaire). Consulting Psychologists Press [Versión española en TEA, Madrid, 1983].

Stiles, W. B. (2001). Assimilation of problematic experiences. Psychotherapy, 38(4), 462-465. https://doi. org/10.1037/0033-3204.38.4.462

Stiles, W. B. (2002). Assimilation of problematic experiences. En J. C. Norcross (ed.), Psychotherapy relationships that work: Therapist contributions and responsiveness to patients (pp. 357-365). Oxford University Press. 
Stiles, W. B. (2007). Theory-building case studies of counselling and psychotherapy. Counselling and Psychotherapy Research, 7(2), 122-127. https://doi.org/10.1080/14733140701356742

Stiles, W. B. (2011). Coming to terms. Psychotherapy Research, 21(4), 367-384. https://doi.org/10.1080/10503 307.2011.582186

Stiles, W. B. y Angus, L. (2001). Qualitative research on clients' assimilation of problematic experiences in psychotherapy. En J. Frommer y D. L. Rennie (eds.), Qualitative psychotherapy research: Methods and methodology (pp. 112-127). Pabst Science Publishers.

Stiles, W. B., Elliott, R., Llewelyn, S. P., Firth-Cozens, J. A., Margison, F. R., Shapiro, D. A. y Hardy, G. (1990). Assimilation of Problematic Experiences by clients in psychotherapy. Psychotherapy, 27(3), 411-420. https://doi.org/10.1037/0033-3204.27.3.411

Stiles., W. B., Morrison, L. A., Haw, S. K., Shapiro, D. A. y Firth-Cozens, J. (1991). Longitudinal Study of Assimilation in Exploratory Psychotherapy. Psychotherapy, 28(2), 195-206. https://doi.org/10.1037/00333204.28.2.195

Vañó, R. y Caro, I. (2015). Sujeto X. Análisis de narraciones a través del modelo de asimilación en la esquizofrenia. Boletín de Psicología, 113, 49-70.

Zigmond, A. S. y Snaith, R. P. (1983). The Hospital Anxiety and Depression Scale. Acta Psychiatrica Scandinavica, 67(6), 361-370. https://doi.org/10.1111/j.1600-0447.1983.tb09716.x 